\title{
24 Months
}

National Cancer Institute

\section{Source}

National Cancer Institute. 24 Months. NCI Thesaurus. Code C156843.

A period of time of twenty-four months. 